\title{
CORRIGENDUM
}

\section{Exosome-associated AAV vector as a robust and convenient neuroscience tool}

E Hudry, C Martin, S Gandhi, B György, DI Scheffer, D Mu, SF Merkel, F Mingozzi, Z Fitzpatrick, H Dimant, M Masek, T Ragan, S Tan, AR Brisson, SH Ramirez, BT Hyman and CA Maguire

Correction to: Gene Therapy (2016) 23, 380-392; doi:10.1038/ gt.2016.11

The initial Figure 2a was erroneously generated from a file from a mouse injected with conventional AAV9-GFP and not exo-AAV9-GFP, as described in the manuscript. We have therefore reformatted this specific panel, and corrected Figure 2a and the figure legend accordingly, now showing an image of the GFP signal detected by 2-photon microscopy after intravenous injection of exoAAV9-GFP in a mouse. The main conclusions of the paper remain unchanged, and both conventional AAV9-GFP and exo-AAV9-GFP mediated detectable in vivo transduction of astrocytes. a

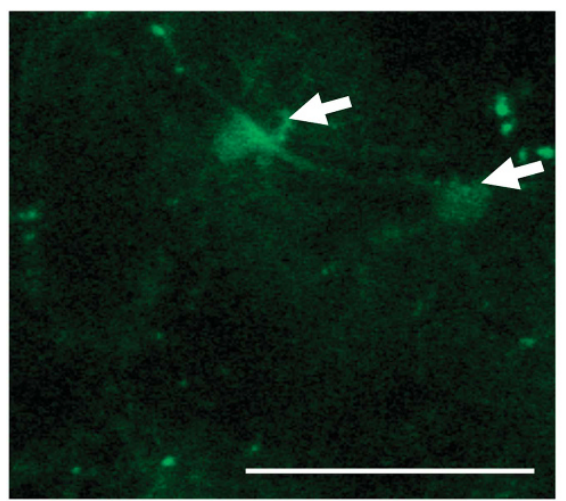

SR101

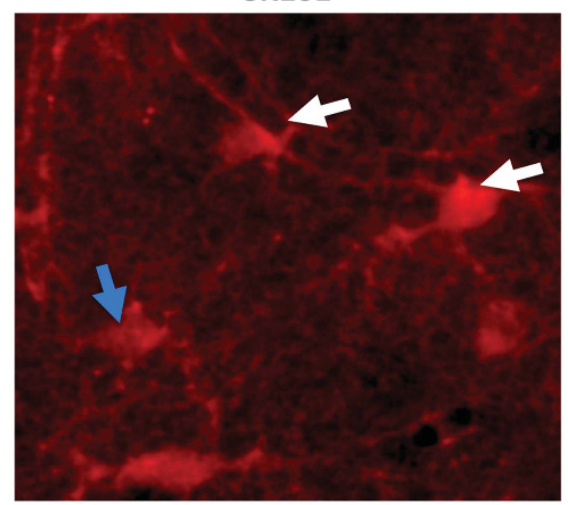

MERGE

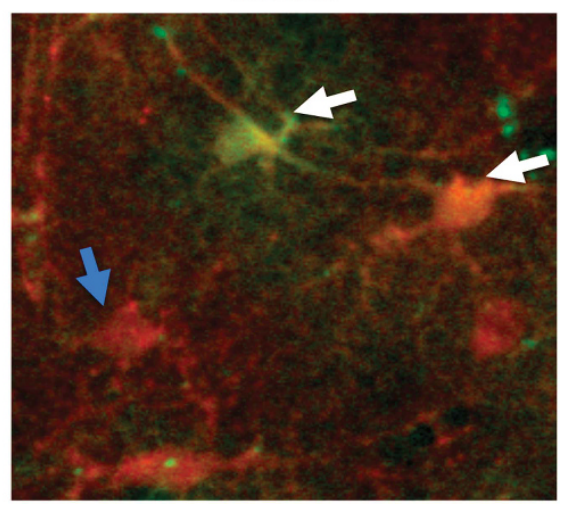

Figure 2. Detection of direct GFP fluorescence by in vivo 2-photon imaging and ex vivo serial 2-photon tomography (STP). BALB/c mice were injected with $7 \times 10^{11}$ g.c. of exo-AAV9-CBA-GFP. After 3 weeks, the GFP fluorescent signal was detected in vivo by multiphoton imaging or ex vivo by STP (in absence of immunostaining). (a) Representative 2-photon images of two GFP-transduced astrocytes (white arrows, identified with the astrocytic marker SR101 topically applied on the brain) in the living animal after cranial window implantation. A nearby astrocyte not expressing detectable GFP is shown (blue arrow). Scale bar: $50 \mu \mathrm{m}$. (b) Three-dimensional reconstruction of the entire cerebellum by postmortem STP tomography imaging, showing the whole vascular tree as well as the direct fluorescent signal across this particular region of the brain. On the right panel, numerous GFP-transduced cells could be identified on a higher magnification image of a small region of the cerebellum. Scale bar: $1000 \mu \mathrm{m}$. (c) GFP signal detected in one section of the cerebellum imaged by 2-photon before three-dimensional reconstruction. Scale bar: $1000 \mu \mathrm{m}$. On the right, two cropped regions of the initial image show GFP-positive astrocytes (yellow arrow), vascular endothelium (blue arrow) and Purkinje cells (purple arrow). Scale bar: $200 \mu \mathrm{m}$. 